\title{
Costs and outcomes associated with hospitalized cancer patients with neutropenic complications: A retrospective study
}

\author{
M. BLANE SCHILLING ${ }^{1}$, CONNIE PARKS ${ }^{1}$ and ROBERT G. DEETER ${ }^{2}$ \\ ${ }^{1}$ Aspen Healthcare Metrics, Centennial, CO 80111; ${ }^{2}$ Amgen Inc., Thousand Oaks, CA 91320, USA
}

Received May 5, 2011; Accepted June 27, 2011

DOI: $10.3892 / \mathrm{etm} .2011 .312$

\begin{abstract}
The average total hospitalization costs for adult cancer patients with neutropenic complications were quantified and the average length of hospital stay (LOS), all-cause mortality during hospitalization and reimbursement rates were determined. This observational retrospective cohort study identified adult patients with cancer who were hospitalized from January 2005 through June 2008 using a large private US health care database (>342 inpatient facilities). ICD-9-CM diagnosis codes identified patients by cancer type and who had neutropenic complications. The utilization and accounting systems of the hospitals were used to calculate mean $( \pm 95 \%$ confidence interval) hospitalization costs and LOS and percent all-cause mortality and reimbursement. Costs were adjusted to 2009 US dollars. There were 3,814 patients who had cancer and neutropenia, 1,809 (47.4\%) also had an infection or fever and $1,188(31.1 \%)$ had infection. Mean hospitalization costs were $\$ 18,042$ (95\% CI 16,997-19,087) for patients with neutropenia, $\$ 22,839$ (95\% CI 21,006-24,672) for patients with neutropenia plus infection or fever and $\$ 27,587$ (95\% CI 24,927-30,247) for patients with neutropenia plus infection. Mean LOS were 9 days (95\% CI 8.7-9.3), 10.7 days (95\% CI 10.2-11.2) and 12.6 days (95\% CI 11.9-13.3), respectively. Mortality followed a similar trend; 8.3, 13.7 and 19.4\%, respectively. By cancer type, hematologic malignancies had the highest average hospitalization costs and longest mean LOS of $\$ 52,579(95 \% \mathrm{CI}$ 42,183-62,975) and 20.3 days (95\% CI 17.4-23.2), and a high mortality rate of $20.0 \%$, while primary breast cancer patients had the lowest cost of $\$ 8,413$ (95\% CI 6,103-10,723), shortest LOS of 5.5 days (95\% CI 4.2-6.8) and lowest mortality (0\%). Mean reimbursement rates were 100.0, 101.5 and $95.4 \%$ for patients with neutropenia, neutropenia plus infection or fever and neutropenia plus infection, respectively. Hospitalized cancer patients with neutropenic complications had a higher all-cause mortality rate and higher inpatient hospitalization
\end{abstract}

Correspondence to: Dr M. Blane Schilling, Aspen Healthcare Metrics, 6300 S. Syracuse Way, Suite 495, Centennial, CO 80111, USA

E-mail: bschilling@medassets.com

Key words: cost, neutropenia, complications costs than those previously published. Results from this study suggest that costs for inpatient hospitalized cancer patients with neutropenic complications are principally reimbursed by payers.

\section{Introduction}

Chemotherapy is the primary treatment for many different types of cancer, including solid cancers and hematologic malignancies. Chemotherapeutic agents lead to potentially life-threatening adverse events, including neutropenia, defined as a below-normal count of neutrophils, which results in impairment of the body's ability to combat infectious pathogens. Severe neutropenia is accompanied by an opportunistic infection that may manifest as a fever in hosts that are capable of mounting a febrile response to infection. In these patients with febrile neutropenia, fever is the first signal of opportunistic infection. Febrile neutropenia often leads to hospitalization, the need for intravenous (i.v.) antibiotics, additional interventional care and further treatment in the outpatient setting $(1,2)$. This may result in chemotherapy dose delays or dose reductions, and interferes with delivery of optimal treatment, adversely affecting patient outcomes, including survival (3-12).

Costs for chemotherapy treatments are significant and costs incurred for treating chemotherapy-associated toxicities contribute to the overall costs of cancer care. In 2007, US cancer costs were estimated to be $\$ 219.2$ billion, with $40 \%$ ( $\$ 89$ billion) attributed to direct medical costs and $60 \%$ (\$130.2 billion) attributed to indirect costs (13). Neutropenic complications resulting from chemotherapy contribute significantly to cancer care costs as part of the treatment for cancer patients. In a study by Caggiano et al, from 1999 data, the incidence of neutropenia-related hospitalizations in the US was estimated to be 60,294 per year, with average costs of $\$ 13,400$ for neutropenia hospitalizations across 13 cancer types (14). Other studies have confirmed that neutropenic complications contribute significantly to the direct and indirect costs of cancer care. Kuderer et al (1), using data from 1995 to 2000, reported average hospitalization costs for febrile neutropenia of $\$ 12,372$ for breast cancer patients, $\$ 18,437$ for lymphoma patients and $\$ 38,583$ for leukemia patients. The most recent data on neutropenia burden of illness are from Weycker et al who used claims data from 2001-2003 (13). In that study, reported average initial hospitalization costs due to neutropenic-related complications (defined as hospitalizations 
with a primary or secondary diagnosis of neutropenia, fever or infection) were $\$ 7,813$ (95\% CI 6,537-9,379) and downstream health care costs, including antibiotic therapy, all hospitalizations and post-discharge outpatient events were $\$ 6,594$ (95\% CI 5,217-8,272).

There are no recent studies estimating total costs associated with neutropenic complications in hospitalized cancer patients. Additionally, no studies to date have assessed the costs and reimbursement in patients with neutropenic complications to evaluate if costs are adequately covered by payers. In this observational, retrospective cohort study, data from a database that included costs from individual inpatient facilities (the providers) were used to calculate the hospitalization costs and outcomes associated with neutropenic complications. The primary objective was to quantify the average total hospitalization costs for cancer patients with neutropenic complications. Secondary objectives were to estimate the average length of hospital stay and mortality rate during hospitalization, and to assess reimbursement rates for hospitalization costs in these patients.

\section{Patients and methods}

Aspen Healthcare Metrics requests data from multiple sources throughout Primary Hospitals and Inpatient Facilities. The collected data are the primary source of information by which Aspen Healthcare Metrics conducts its consulting business. Once the collected data are accepted, they are formatted, processed and stored in a proprietary database known as the Navigator database. The data are then made available for established reporting and ad hoc purposes, as well as benchmarking.

Data. The collected data, specifically received from Materials Management, Decision Support, Finance/Accounting, Case Management and Pharmacy, among others, is allocated into spend and patient segments. The spend data primarily consists of closed receipt files (purchase orders/invoices), while the patient data primarily consist of demographics (UB-04), diagnosis and procedure codes, detailed charges and facilityspecific charge masters. The data received undergo multiple levels of quality assurance and quality control checks, both automated and manual. The check process may identify data containing irresolvable issues, which require a resubmission pending resolution of identified issues.

Processing. Spend data processing is facilitated by Analysts utilizing supply item descriptions and catalog numbers to categorize spend. The spend data contain purchase order and invoice data, manufacturer and vendor data, quantity and price data. The description, manufacturer and vendor data are normalized, allowing for unique categorization of the items. The associated spend data may then be aggregated into unique categories, or into super-sets of more general categories, such as implant, laboratory, ancillary, pharmacy and medical/ surgical costs. Patient billing identifiers, physician identifiers and other data may also be associated with the items, allowing for trend analysis. The preliminary spend data are summarized and then reviewed by Consultants through a spend data update process identifying further spend enhancement opportunities based on their subject matter expertise and market knowledge.
Once the spend data updates are applied, it is finalized in the Navigator database.

Patient data processing is also facilitated by Analysts, utilizing a unique patient billing identifier to associate diagnosis and procedure codes, as well as expected reimbursement. The procedure codes are then used to associate a physician and physician group, if applicable. The patient billing identifier is also associated with charge codes, including service date, units and total charges. The charge codes, which uniquely identify utilized items, are then associated with revenue codes (UB-92) and Current Procedural Terminology, Fourth Edition (CPT-4)/ Healthcare Common Procedure Coding System (HCPCS) codes in a facility-specific charge master. The charge master contains facility-specific charges, total, variable and fixed costs for one unit of each item, allowing for utilization analysis. In the event that the facility does not store cost information in the charge master, total costs are allocated at the item level using a ratio of cost to charge based on the facility's Medicare Cost Report Worksheet C, Part I. The total cost is then broken down between variable (directly related to patient care) and fixed (relatively static overhead) costs based upon Aspen Healthcare Metric's experience. The preliminary patient data are then blended with the finalized spend data, merging price and cost, and reviewed by Consultants through a patient data update process, again identifying further cost enhancements based on their subject matter expertise and market knowledge. Once the patient data updates are applied, it is finalized in the Navigator database.

Database. The Navigator database contains information from more than 342 clinical providers across various geographic regions of the US, and has over 300 million items of hospital utilization data from approximately 11 million patients. Specific data available in the database include patient demographics, costs related to inpatient, outpatient, ambulatory surgery and pharmaceuticals, and all-payer data, including Medicare Severity Diagnosis Related Groups (MS-DRG) as well as International Classification of Diseases, Ninth Revision, Clinical Modification (ICD-9-CM) diagnosis and procedure codes.

For purposes of this study, data included in the Navigator database were de-identified to preserve patient anonymity and confidentiality, to comply with the Health Insurance Portability and Accountability Act of 1996 (15) and Federal Guidelines on Public Welfare and the Protection of Human Subjects (16). Additionally, data drawn from the Navigator database have been previously scrutinized for accuracy during presentation to Aspen Healthcare Metric's customers, and are believed to be accurate in quantifying cost and outcomes. The data used in this study were therefore considered valid for use in estimating hospitalization costs.

Patients. The study population included inpatients $\geq 18$ years of age who had at least 1 ICD-9-CM diagnosis code for malignant neoplasm and a diagnosis code for neutropenia, or neutropenia with infection or fever, and whose records were captured in the Navigator database (Table I). The study was limited to patients discharged between January 2005 and June 2008. Since no single ICD-9-CM code for cancer-related neutropenic complications exists, a combination of ICD-9-CM diagnoses 
Table I. Inclusion/exclusion criteria used to identify hospitalized cancer patients with neutropenic complications.

Inclusion criteria:

Inpatients discharged between January 2005 and June 2008.

Were 18 years of age or older.

Had at least one diagnosis code (primary or secondary) for malignant neoplasm (ICD-9-CM code 140.xx-208.xx) and neutropenia (ICD-9-CM code 288.0).

Four groups of patients with neutropenic complications were identified as follows:

Neutropenia: ICD-9-CM code 288.0.

Neutropenia plus infection or fever: ICD-9-CM code 288.0 and ICD-9-CM codes for opportunistic infection or ICD-9-CM code 288.0 and ICD-9-CM code for fever of unknown origin (780.6).

Neutropenia without infection or fever: ICD-9-CM code 288.0 and no ICD-9-CM codes for opportunistic infection or ICD-9-CM code 288.0 and no ICD-9-CM code for fever of unknown origin (780.6).

Neutropenia plus infection: ICD-9-CM code 288.0 and ICD-9-CM codes for opportunistic infection.

\section{Exclusion criteria:}

Had a UB-92 discharge status of 30 (still inpatient or expected to return as an outpatient) or 99 (unknown).

Had undergone a bone marrow or stem cell transplant (ICD-9-CM codes 41.00-41.09, V42.81 and V42.82 or

CPT codes 38204-38242).

Had a diagnosis of HIV (ICD-9-CM code 042 or V08).

ICD-9-CM, International Classification of Diseases, Ninth Revision, Clinical Modification. UB-92, Health Care Financing Administration Uniform Bill-92.

codes was used to identify cancer patients with i) neutropenia, ii) neutropenia plus infection or fever, iii) neutropenia without infection or fever and iv) neutropenia plus infection (summarized in Table I). This method has been used in other studies $(13,17)$. Patients were excluded if they were still hospitalized or were expected to return as an outpatient, had undergone a bone marrow or stem cell transplant, or had a diagnosis code for HIV (Table I). No institutional review board approvals were required, since this was an observational, retrospective cohort study.

Study measures. In this study, the economic impact of hospitalizations for neutropenic complications was determined by calculating average total inpatient hospital costs per admission from clinical and billing records. Information on the utilization of health care resources and actual costs (not charges) were used.

Utilization of inpatient hospital resources was categorized according to the following groups: critical care, routine care, diagnostics, EKG/ECG, laboratory and blood tests, medical, surgical and sterile supplies, occupational therapy, and pharmacy and intravenous supplies. Costs were derived from the actual invoiced costs of the items to the inpatient facility. Inputs included unit variable costs, unit fixed costs, unit total costs (e.g., pharmaceutical product costs were provided from pharmaceutical distributor reports that detail exact price paid), room and services, and other department costs. Physician fees were not captured in the database.

Patient demographics and other treatment-related variables collected include gender, age, length of stay (defined by date of admission and date of discharge), admission source and discharge status. Co-morbid health conditions, identified using ICD-9-CM codes, were also collected.
Endpoints. The primary endpoint was average hospitalization costs per admission. Secondary endpoints included length of hospital stay, number of deaths by any cause during hospitalization and hospitalization costs by cancer type. Also, average payer reimbursement was calculated from all-payer data obtained from the cost accounting systems of the facilities represented by these patients and the percentage of inpatient hospitalization costs vs. the reimbursement was determined.

Statistical analysis. No formal hypothesis was tested, since the primary objective of the study was to estimate total costs. Descriptive statistics, including both average hospital admission costs and average reimbursement, were calculated. The analyses for this study were performed using T-SQL commands within a 2002 SQL Server database. Microsoft Excel 2007 was used to calculate confidence intervals (CIs). The average hospitalization costs per admission across sites with corresponding 95\% CIs are presented. Mean hospital costs per admission were adjusted to 2009 costs by applying a $3 \%$ annual inflation rate, based on consensus guidelines for conducting cost-effectiveness analyses published by the Panel on Cost-effectiveness in Health and Medicine (18). Admission and discharge dates were collected and used to calculate average length of hospital stay and corresponding 95\% CIs. Mortality, defined as the number of patient deaths from all causes during the hospitalization for neutropenic complications, was determined. For the primary analysis, as per pre-defined exclusion criteria, patients who died within 1 day of admission were excluded from the hospitalization costs, length of hospital stay and reimbursement calculations because their data would not accurately reflect the cost of caring for patients with this disease state. Subgroup analyses on average total hospitalization costs, length of hospital stay and all-cause 


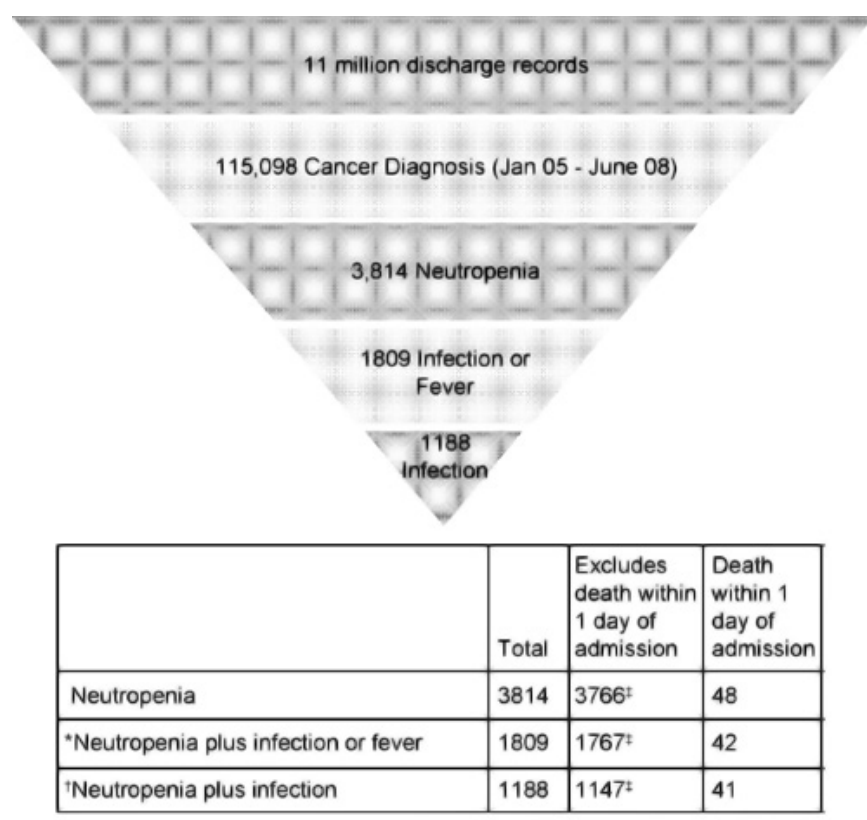

Figure 1. Patient populations analyzed. "Broad definition of neutropenia and infections. "Main definition of neutropenia and infections. "Population analyzed for hospitalization costs and length of hospital stay.

mortality stratified by cancer type, including hematologic malignancies [excluding non-Hodgkin's lymphoma (NHL)], lung or bronchial or breast cancer, were also performed. In addition, average reimbursement was calculated from allpayer data contained in the database and the proportion of reimbursed hospitalization costs was determined.

Sensitivity analyses were conducted on overall cost, length of stay and reimbursement calculations, excluding patients with hematologic malignancies (excluding NHL), since neutropenia could be disease related and not causally related to chemotherapy. An additional sensitivity analysis was conducted, including patients who died within 1 day of hospital admission.

\section{Results}

Patients. The Navigator database contained over 11 million admission records for patients who were discharged during the period of January 2005 to June 2008 (Fig. 1). The number of cancer patients hospitalized with a primary or secondary diagnosis of neutropenia identified from these records was 3,814 . Of the patients with cancer and neutropenia, a subset of 1,809 had neutropenia plus infection or fever, and an additional subset of 1,188 had neutropenia plus infection (Fig. 1).

Demographic data are presented for the 1,188 hospitalized cancer patients with neutropenia plus infection (Table II). The patient population was $53.6 \%$ male and $53 \%$ were $\geq 65$ years of age. The most common primary cancer types identified in this population were NHL (15.4\%), other hematologic (11.4\%), lung or bronchial (8.3\%), metastatic breast (3.4\%) and primary breast (3.1\%) cancer. The most common co-morbid conditions were electrolyte imbalance (45.4\%), acute pulmonary disease (44.0\%), heart disease (40.5\%), anemia (37.4\%), hypertensive disease $(34.6 \%)$, cardiac dysrhythmias $(22.3 \%)$ and chronic pulmonary disease $(19.0 \%)$.
Table II. Patient demographic and clinical characteristics.

Patients with neutropenia plus infection ${ }^{\mathrm{a}, \mathrm{b}}$

$(\mathrm{n}=1,188)$

Female, n (\%)

$551(46.4)$

Age (years), median (range)

$65(18-100)$

$<65$ years, $\mathrm{n}(\%)$

$558(47.0)$

Cancer type, $\mathrm{n}(\%)$

NHL

$183(15.4)$

Lung and bronchial

$99(8.3)$

Metastatic breast

$40 \quad(3.4)$

Primary breast

$37 \quad(3.1)$

Other hematologic cancers ${ }^{\mathrm{c}}$

$135(11.4)$

History of co-morbidity, n (\%)

$1,111(93.5)$

$539(45.4)$

$523(44.0)$

$481(40.5)$

$444(37.4)$

$411(34.6)$

$265(22.3)$

$226(19.0)$

$207(17.4)$

$192(16.2)$

$160(13.5)$

$135(11.4)$

$30(2.5)$

15 (1.3)

Acute myocardial infarction

(initial episode)

${ }^{\mathrm{a}}$ Main definition of neutropenic complications. ${ }^{\mathrm{b}}$ Includes patients who died within 1 day of admission. 'Includes myeloid leukemia, monocytic leukemia, other leukemia and malignant neoplasm of connective and other soft tissue. NHL, non-Hodgkin's lymphoma.

Overall costs and outcomes associated with hospitalized cancer patients with neutropenic complications. Mean hospitalization costs were $\$ 18,042(95 \%$ CI 6.997-19,087) for cancer patients with neutropenia, $\$ 22,839$ (95\% CI 21,006-24,672) for cancer patients with neutropenia plus infection or fever, and $\$ 27,587(95 \%$ CI $24,927-30,247)$ for cancer patients with neutropenia plus infection (Fig. 2A). The average length of hospital stay followed a directional pattern consistent with that for the hospitalization costs; 9.0 (95\% CI 8.7-9.3), 10.7 (95\% CI 10.2-11.2) and 12.6 (95\% CI 11.9-13.3) days, respectively (Fig. 2B). Frequency of death from any cause during hospitalization also followed a similar trend; 8.3, 13.7 and 19.4\%, respectively (Fig. 2C).

Costs and outcomes by cancer type associated with hospitalized cancer patients with neutropenic complications. In cancer patients hospitalized with neutropenia plus infection $(\mathrm{n}=1,147)$, the most common primary cancer types were NHL (20.8\%, 178 patients), other hematologic malignancies (15.5\%, 132 patients), lung or bronchial (8.4\%, 96 patients), metastatic breast $(3.2 \%, 37$ patients) and primary breast (3.2\%, 37 patients) cancer. Mean length of hospital stay was 
A

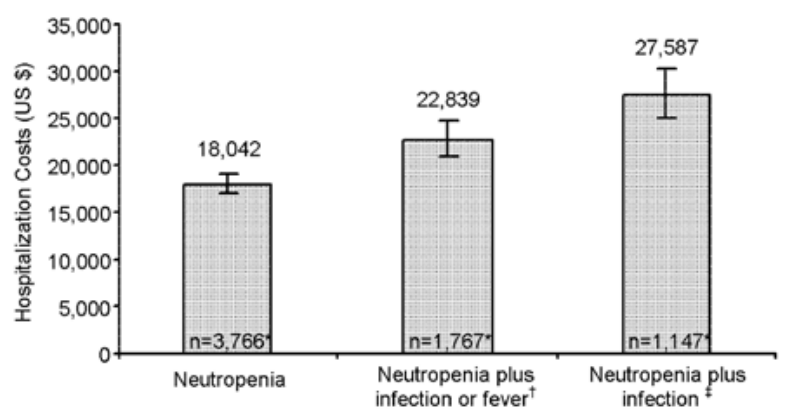

B

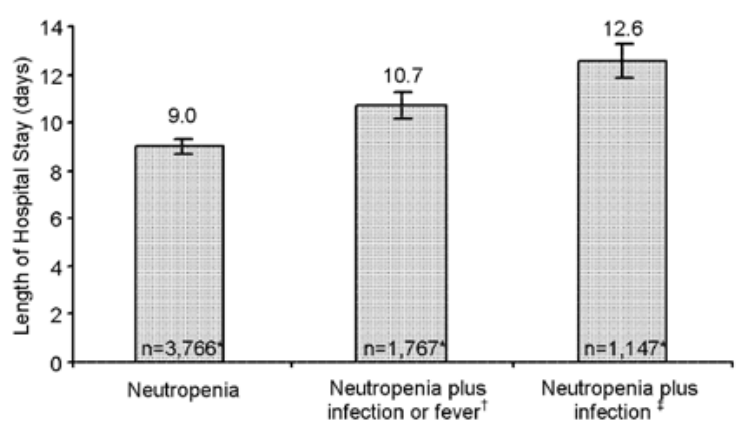

C

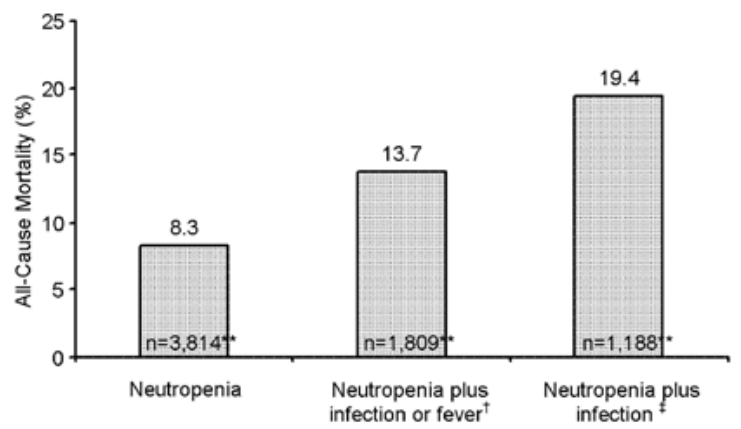

Figure 2. Costs and outcomes associated with hospitalized cancer patients with neutropenia and infections. (A) Hospitalization costs [US\$, mean $( \pm 95 \% \mathrm{CI})]$. (B) Length of hospital stay [days, mean $( \pm 95 \% \mathrm{CI})]$. (C) Allcause mortality (\%). "Excludes patients who died within 1 day of admission. Broad definition of neutropenia and infections. ${ }^{\dagger}$ Main definition of neutropenia and infections. ${ }^{* *}$ Includes patients who died within 1 day of admission. US\$, United States dollars.

12.0 (95\% CI 10.3-13.7), 20.3 (95\% CI 17.4-23.2), 9.5 (95\% CI 7.5-11.5), 7.7 (95\% CI 6.2-9.2) and 5.5 (95\% CI 4.2-6.8) days, respectively (Fig. 3B). All-cause mortality during hospitalization $(\mathrm{n}=1,188)$ was $20.0 \%$ for other hematologic malignancies, $19.7 \%$ for NHL, $21.2 \%$ for lung or bronchial cancer and $20.0 \%$ for metastatic breast cancer (Fig. 3C). No patients with primary breast cancer died during hospitalization (Fig. 3C).

In a subgroup analysis by cancer type (Fig. 3A), other hematologic malignancies had the highest average inpatient financial costs of $\$ 52,579$ (95\% CI 42,183-62,975) followed by NHL $(\$ 26,208,95 \%$ CI $21,202-31,214)$, lung or bronchial cancer $(\$ 17,382,95 \%$ CI $13,178-21,586)$, metastatic breast cancer $(\$ 14,341,95 \%$ CI $10,830-17,852)$ and primary breast cancer $(\$ 8,413,95 \%$ CI $6,103-10,723)$. Patients with other cancers $(\mathrm{n}=507)$ were excluded from this subgroup analysis because the number of patients per specific cancer were low
A

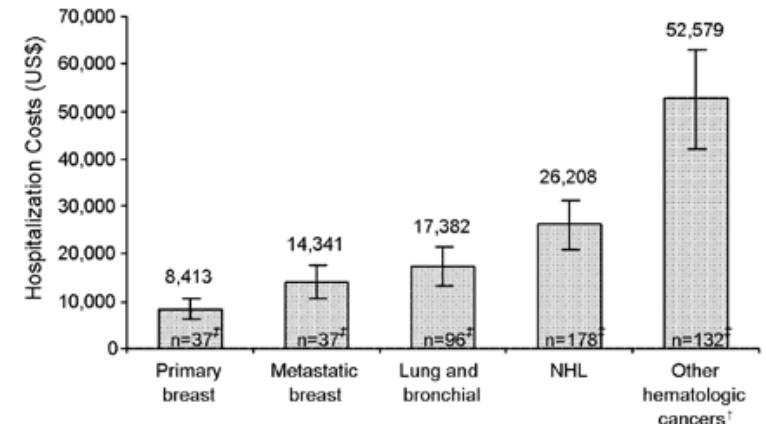

B
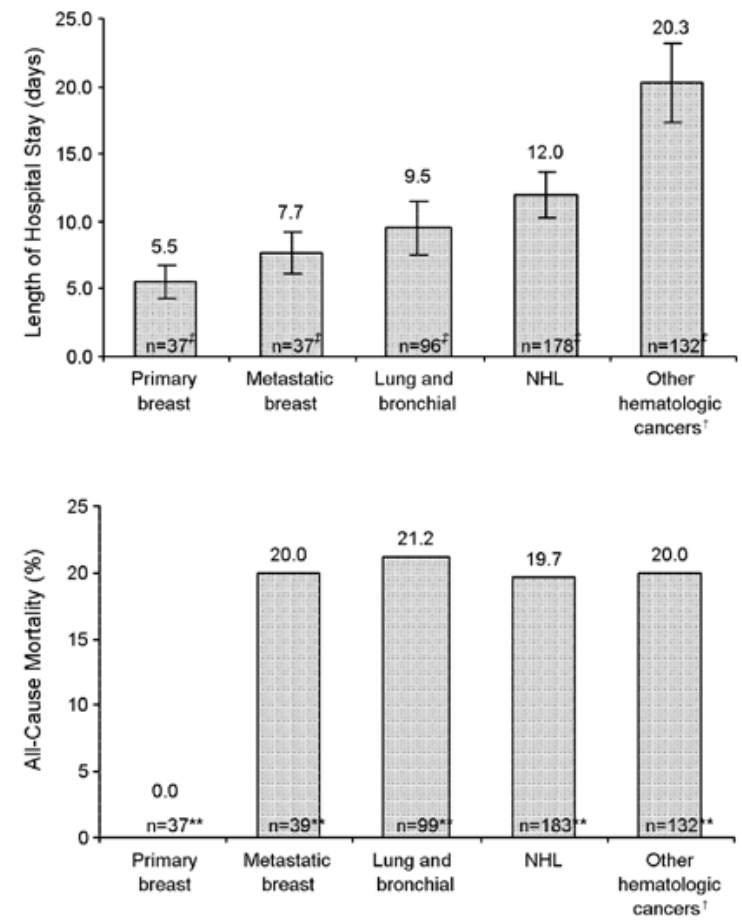

Figure 3. Costs and outcomes by cancer type associated with hospitalized cancer patients with neutropenia and infections. (A) Hospitalization costs [US\$, mean $( \pm 95 \% \mathrm{CI})$ ]. (B) Length of hospital stay [days, mean $( \pm 95 \% \mathrm{CI})$ ] (C) All cause mortality (\%). "Main definition of neutropenia and infections in this study. ${ }^{\dagger}$ Patients with other cancers $(n=507)$ were excluded from this analysis. ${ }^{+E x c l u d e s ~ p a t i e n t s ~ w h o ~ d i e d ~ w i t h i n ~} 1$ day of admission. ${ }^{* *}$ Includes patients who died within 1 day of admission. NHL, non-Hodgkin's lymphoma; US\$, United States dollars.

and treatments were too variable to generalize costs in a relevant manner.

Reimbursement of costs associated with hospitalized cancer patients with neutropenic complications. Analysis of average inpatient hospitalization costs and average reimbursed costs for cancer patients hospitalized with neutropenic complications shows that hospitalization costs were principally reimbursed by payers (Table III). Mean reimbursement rates of inpatient hospitalization costs for cancer patients with neutropenia were $100 \%$, and $101.5 \%$ for patients with neutropenia plus infection or fever, with an average difference between average hospitalization costs and average reimbursements of $\$ 11(95 \% \mathrm{CI}$ $-1,051$ to 1,073$)$ and $\$ 353(95 \%$ CI $-1,632$ to 2,338$)$, respectively. Inpatient hospitalization costs in cancer patients with 
Table III. Costs and reimbursement associated with hospitalized cancer patients with neutropenic complications.

\begin{tabular}{|c|c|c|c|c|}
\hline & $\begin{array}{c}\text { Neutropenia }{ }^{a, b} \\
\quad(n=3,766)\end{array}$ & $\begin{array}{l}\text { Neutropenia plus } \\
\text { infection or fever } \text { a }^{\mathrm{a}, \mathrm{c}} \\
(\mathrm{n}=1,767)\end{array}$ & $\begin{array}{l}\text { Neutropenia without } \\
\text { infection or fever }{ }^{\mathrm{a}, \mathrm{c}} \\
(\mathrm{n}=1,999)\end{array}$ & $\begin{array}{c}\text { Neutropenia plus } \\
\text { infection }{ }^{\mathrm{a}, \mathrm{d}} \\
(\mathrm{n}=1,147)\end{array}$ \\
\hline $\begin{array}{l}\text { Hospitalization costs, mean (95\% CI) } \\
\text { US\$ }\end{array}$ & $\begin{array}{c}18,042 \\
(16,997-19,087)\end{array}$ & $\begin{array}{c}22,839 \\
(21,006-24,672)\end{array}$ & $\begin{array}{c}13,801 \\
(12,716-14,887)\end{array}$ & $\begin{array}{c}27,587 \\
(24,927-30,247)\end{array}$ \\
\hline $\begin{array}{l}\text { Hospital reimbursements, mean }(95 \% \mathrm{CI}) \\
\text { US\$ }\end{array}$ & $\begin{array}{c}18,052 \\
(16,769-19,335)\end{array}$ & $\begin{array}{c}23,191 \\
(20,936-25,446)\end{array}$ & $\begin{array}{c}13,367 \\
(12,067-14,667\end{array}$ & $\begin{array}{c}26,321 \\
(23,165-29,478)\end{array}$ \\
\hline $\begin{array}{l}\text { Difference (loss) (95\% CI), } \\
\text { US\$ }\end{array}$ & $\begin{array}{c}11 \\
(-1,051 \text { to } 1,073)\end{array}$ & $\begin{array}{c}353 \\
(-1,632 \text { to } 2,338)\end{array}$ & $\begin{array}{c}435 \\
(-1,356 \text { to } 487)\end{array}$ & $\begin{array}{c}1,266 \\
(-4,110 \text { to } 1,578)\end{array}$ \\
\hline $\begin{array}{l}\text { Proportion of hospitalization costs } \\
\text { reimbursed }(\%)\end{array}$ & 100.1 & 101.5 & 96.9 & 95.4 \\
\hline
\end{tabular}

${ }^{a}$ Excludes patients who died within 1 day of admission. ${ }^{b}$ Includes patients with neutropenia plus infection or fever, patients with neutropenia plus infection and patients with neutropenia without infection or without fever. ${ }^{c}$ These patients are a subset of the patients with neutropenia. ${ }^{\mathrm{d}}$ These patients are a subset of the patients with neutropenia plus infection or fever.

neutropenia plus infection were reimbursed at an average of $95.4 \%$, with an average difference of $\$ 1,266$ (95\% CI -1,578 to 4,110$)$.

Sensitivity analyses. Since neutropenia could be disease-related and not causally related to chemotherapy treatment, sensitivity analyses that excluded patients with other hematologic malignancies (but included NHL) showed slightly reduced overall costs $(\$ 14,891, \$ 18,653$ and $\$ 22,981)$ and length of stay (7.9, 9.2 and 10.9 days), and similar mortality (8.0,13.6 and 19.9\%) for cancer patients with neutropenia, neutropenia plus infection or fever, and neutropenia plus infection, respectively. In addition, patients who died within 1 day of admission were excluded from the hospitalization costs, length of hospital stay and reimbursement calculations because their data would not accurately reflect the cost of caring for patients with this disease state. Including these patients into these analyses (data not shown), did not significantly alter the results. Further investigation of these costs indicated that costs were primarily related to morgue and other non-disease related costs, some of them significant (e.g., cause of death investigation).

\section{Discussion}

Results from this retrospective observational study show high average inpatient hospitalization costs for cancer patients with neutropenia $(\$ 18,042)$, neutropenia plus infection or fever $(\$ 22,839)$, and neutropenia plus infection (\$27,587) (Fig. 2A). These average hospitalization costs are higher than those previously reported $(1,13,14)$. A study by Caggiano et al, using 1999 data from a longitudinal hospital discharge database that contained data from across seven US states, reported average neutropenia hospitalization costs (SD) of $\$ 13,400$ $(\$ 21,000)$ across 13 cancer types (14). A study by Kuderer et al, using a longitudinal hospital discharge database with data from 115 US academic medical centers collected over 6 years (1995 to 2000), reported average febrile neutropenia hospitalization costs of $\$ 19,110$ (1). A recent study by Weycker et al, using 2001 to 2003 data, reported average neutropenia- related hospitalization costs of $\$ 7,813$ (95\% CI 6,537-9,379) (13). However, when downstream health care costs, including antibiotic therapy, hospitalizations and post-discharge outpatient events were considered, additional costs of $\$ 6,594$ (95\% CI 5,217-8,272) were observed (13). The authors of that study concluded that prior research focusing on initial hospitalizations only may have underestimated the total costs of neutropenia complications by as much as $40 \%$ (13). Reasons behind the higher hospitalization costs in our study compared to previous studies may include inflationary price increases, changes in technology, differences in sampled populations, databases used and treatments provided to patients.

When analyzed by cancer type, hematologic malignancies (excluding NHL) had the highest average inpatient financial costs of $\$ 52,579$, followed by NHL $(\$ 26,208)$, lung or bronchial cancer $(\$ 17,382)$, metastatic breast cancer $(\$ 14,341)$ and primary breast cancer $(\$ 8,413)$ (Fig. 3A). Earlier studies reported a similar pattern $(1,14)$. Caggiano et al $(14)$ reported higher average neutropenia-related hospitalization costs for patients with NHL $(\$ 11,600)$ or leukemia $(\$ 28,200)$ than for those with breast cancer $(\$ 7,100)$. Kuderer et al reported average febrile neutropenia-related hospitalization costs of $\$ 18,437$ for patients with lymphoma, $\$ 38,583$ for patients with leukemia and $\$ 12,372$ for patients with breast cancer (1). Findings for length of hospital stay appear to support this pattern [Fig. 3B in our study and (14)], with longer hospital stays for patients with hematologic malignancies than for patients with other cancer types.

The administrative database utilized in our study identifies specific utilization of health care resources down to the item level and associated costs obtained from the supply costs files linked to billing claims and reimbursement. Thus, the database has an advantage of providing information comparing hospitalization costs vs. reimbursed charges. Even though inpatient hospitalization costs for cancer patients with neutropenic infections were principally reimbursed, our data suggest that these costs per tumor may vary. When hematologic malignancies (excluding NHL) are excluded from the overall reimbursement analysis, average reimbursement rates 
of inpatient hospitalization costs were $88 \%$ for patients with neutropenia, $89 \%$ for patients with neutropenia plus infection or fever, and $84 \%$ for patients with neutropenia plus infection. These reimbursement rates are lower than those seen when hematologic malignancies (excluding NHL) are included in the overall analysis (Table III).

The findings from our study suggest that inpatient facilities and payers carry the financial costs of managing hospitalizations for cancer patients with neutropenic complications. Facilities and payers should consider margin management through clinical alterations aimed at decreasing costs while maintaining quality of care. Furthermore, improvements in future treatment of cancer patients with neutropenic complications may reduce mortality and morbidity, but likely at a higher cost to payers and providers. In general, hospitals break even or lose small percentages of revenue. Mostly, payers cover hospitalization costs for the care of cancer patients with neutropenia and infections, and therefore have the greatest stake in understanding costs.

In an era with tight constraints on payer budgets and particularly government agencies, efforts should be made to find the most cost-effective disease prevention and treatment programs. Decisions by many government payers already dictate treatment and reimbursement in high-cost disease settings (19). Private payers continually monitor these decisions by government payers and often adopt them. In fact, several conditions that are not completely preventable and occur even during high-quality medical care are no longer reimbursed by the Center for Medicare and Medicaid Services (19) (i.e., surgical site infections following certain elective procedures, certain manifestations of poorly controlled blood sugar levels, deep vein thrombosis or pulmonary embolism following total knee and hip replacement procedures and stage III and IV decubitus ulcers). A number of public and private sector payers have also decided not to reimburse hospitals for health care that follows from these events (19). If neutropenic complications were to be considered preventable illnesses, given the financial constraints of the current environment, there is a possibility that reimbursement for these conditions could become restricted. Hospitals would have no way of obtaining reimbursement for conditions arising as a result of care provided by unaffiliated facilities and physicians. Understanding the financial costs of managing hospitalizations for cancer patients with neutropenic complications would be an important issue with potentially significant fiscal implications under this framework.

Effective methods for preventing neutropenic complications include the use of primary prophylaxis with growth factors during chemotherapy; however, the costs for prevention are also high (8,20-24). Costs and outcomes data could be used to compare the overall costs and benefits of prophylactic use of growth factors vs. reactive treatment in hospital facilities. Results from this study, along with other decision-making tools, may help inform decision-makers regarding the most cost-effective ways for managing cancer patients who are at risk for acquiring neutropenic complications.

Limitations. There are limitations associated with observational and retrospective studies of hospital costs using data from an administrative database, including errors in coding.
The foremost limitation of the present study is the inability to link any of these costs (including infections), specifically to neutropenic complications or the chemotherapy that may have contributed to the neutropenic complication. Since no ICD-9-CM code exists for the clinical manifestation of neutropenic complications, this study used a combination of codes for neutropenia, fever and infections. With this nonclinically specific method, a possibility exists that coding of neutropenia-related events may not be reliably or uniformly documented across practices. However, similar issues and resolution methods have been used in other claims-based studies $(13,17)$.

The analysis of health care costs in this study is exploratory and descriptive in nature. Patient clinical and treatment covariates, including pre-existing conditions, patient histories, chemotherapy treatment regimens, diagnostic test results and cause of death, were not available. This potentially leads to incomplete or biased assessment of costs. In addition, this also limits testing of associations of neutropenic complications with several factors of interest, including chemotherapy administration (chemotherapy-induced neutropenia), chemotherapy regimen cycle and cause of death during hospitalization.

Since the Aspen Healthcare Metrics Navigator database only captures institution-specific costs, physician fees were not captured and as a result, total costs of care are therefore underestimated. Finally, the study results are only generalizable to patients at inpatient facilities (Aspen Healthcare Metrics clients) of particular geographic regions and may not be generalizable to patients in other settings.

In conclusion, cancer patients with neutropenic complications are associated with high inpatient hospitalization costs that exceed those previously published. All-cause mortality during hospitalization is also high. Results from this study suggest that costs for inpatient hospitalized cancer patients with neutropenic complications are principally reimbursed by payers.

\section{Acknowledgements}

Funding for this study was provided by the Amgen Inc. The assistance of Martha Mutomba, PhD, of Amgen Inc., and Julie Gage of Gage Medical Writing with preparation of the manuscript is acknowledged.

\section{References}

1. Kuderer NM, Dale DC, Crawford J, et al: Mortality, morbidity, and cost associated with febrile neutropenia in adult cancer patients. Cancer 106: 2258-2266, 2006.

2. Link BK, Budd GT, Scott S, et al: Delivering adjuvant chemotherapy to women with early-stage breast carcinoma: current patterns of care. Cancer 92: 1354-1367, 2001.

3. Adida C, Haioun C, Gaulard P, et al: Prognostic significance of surviving expression in diffuse large B-cell lymphomas. Blood 96: 1921-1925, 2000.

4. Lyman GH, Dale DC and Crawford J: Incidence and predictors of low dose-intensity in adjuvant breast cancer chemotherapy: a nationwide study of community practices. J Clin Oncol 21: 4524-4531, 2003.

5. Hryniuk WM: Average relative dose intensity and the impact on design of clinical trials. Semin Oncol 14: 65-74, 1987.

6. Klastersky J, Paesmans M, Rubenstein EB, et al: The Multinational Association for Supportive Care in Cancer risk index: a multinational scoring system for identifying low-risk febrile neutropenic cancer patients. J Clin Oncol 18: 3038-3045, 2000 . 
7. Talcott JA, Siegel RD, Finberg R and Goldman L: Risk assessment in cancer patients with fever and neutropenia: a prospective, twocenter validation of a prediction rule. J Clin Oncol 110: 316-322, 1992.

8. Smith TJ, Khatcheressian J, Lyman GH, et al: 2006 update of recommendations for the use of white blood cell growth factors: an evidence-based clinical practice guideline. J Clin Oncol 24: 3187-3205, 2006

9. Bonadonna G, Moliterni A, Zambetti M, et al: 30 years' follow up of randomized studies of adjuvant CMF in operable breast cancer: cohort study. BMJ 330: 217, 2005.

10. Epelbaum R, Faraggi D, Ben-Arie Y, et al: Survival of diffuse large cell lymphoma. A multivariate analysis including dose intensity variables. Cancer 66: 1124-1129, 1990.

11. Kwak LW, Halpern J, Olshen RA and Horning SJ: Prognostic significance of actual dose intensity in diffuse large-cell lymphoma: results of a tree-structured survival analysis. J Clin Oncol 8: 963-977, 1990.

12. Lepage E, Gisselbrecht C, Haioun C, et al: Prognostic significance of received relative dose intensity in non-Hodgkin's lymphoma patients: application to LNH-87 protocol. The GELA (Groupe d'Etude des Lymphomes de l'Adulte). Ann Oncol 4: 651-656, 1993.

13. Weycker D, Malin J, Edelsberg J, Glass A, Gokhale M and Oster G: Cost of neutropenic complications of chemotherapy. Ann Oncol 19: 454-460, 2008.

14. Caggiano V, Weiss RV, Rickert TS and Linde-Zwirble WT: Incidence, cost, and mortality of neutropenia hospitalization associated with chemotherapy. Cancer 103: 1916-1924, 2005.

15. Health Insurance Portability and Accountability Act of 1996, 42 USC, pp1320-1322, 1996.

16. US Dept of Health and Human Services: Public WelfareProtection of Human Subjects, 45 CFR 46. www.hhs.gov/ohrp/ humansubjects/guidance/45cfr46.htm. Accessed Aug 12, 2009.

17. Weycker D, Malin J, Kim J, et al: Risk of hospitalization for neutropenic complications of chemotherapy in patients with primary solid tumors receiving pegfilgrastim or filgrastim prophylaxis: a retrospective cohort study. Clin Ther 31: 1069-1081, 2009.
18. Gold M, Siegel JE, Russell LB and Weinstein MC: CostEffectiveness in Health and Medicine. Oxford University Press, New York, pp91-92, 1996.

19. Center for Medicare and Medicaid Services: Eliminating serious, preventable, and costly medical errors-never events, CMS Fact Sheet, May, 18, 2006. www.cms.hhs.gov/apps/media/press/ factsheet.asp? Counter $=1863 \&$ int NumPerPage $=10 \&$ checkDate $=$ $\&$ checkKey $=\&$ srchType $=1 \&$ numDays $=3500 \&$ srchOpt $=0 \&$ srch Data $=\&$ keywordType $=$ All \&chkNewsType $=6 \&$ intPage $=\&$ show All $=\& p$ Year $=\&$ year $=\&$ desc $=$ false $\&$ cboOrder $=$ date. Accessed Oct 5, 2009.

20. National Comprehensive Cancer Network clinical practice guidelines in oncology: Myeloid growth factors in cancer treatment, version 2.2005 [online]. www.ncen.org/professionals/ physician_gls/PDF/myeloid_growth.pdf.2005. Accessed Mar 12, 2009.

21. Glaspy J, Hackett J, Flyer P, et al: Febrile neutropenia is associated with an increase in the incidence, duration, and severity of chemotherapy toxicities. Blood 98: 432b, 2001.

22. Brown RE, Hutton J and Burrell A: Cost effectiveness of treatment options in advanced breast cancer in the UK. Pharmacoeconomics 19: 1091-1102, 2001.

23. Fortner BV, Stolshek B, Tauer KW, et al: Final analysis: chemotherapy-induced neutropenia (CIN) is associated with lower quality of life (QoL) in patients (pts) with cancer. Ann Oncol 13 (Suppl 15): 174, 2002.

24. Okon TA, Fortner BV, Schwartzberg L, et al: Quality of life (QOL) in patients with grade IV chemotherapy-induced neutropenia (CIN). Proc Am Soc Clin Oncol 21: 275b, 2002. 